6 Billingsley P. Convergence of Probability Measures. New York: J Wiley and Sons, 1968

7 王梓坤. 随机过程通论. 北京: 北京师范大学出版社, 1996

(1997- 12-08 收稿)

\title{
Hopf 代数的扭曲积和量子偶
}

胡国权

(中山大学数学系, 广州 510275)

摘要 设 $\sigma$ 是 $\mathrm{Hopf}$ 代数对 $(B, H)$ 上一个斜配对, $A$ 是左 $(B, H)$ 双余模代数, 利用 $\sigma$ 和 $B, H$ 在 $A$ 上的余作用改变 $A$ 的乘法得到一个新的代数 $A$ o, 称为 $A$ 的扭曲积. 证明 $\sigma$ 诱导 $(B \leftarrow$ $\left.B^{\text {cop }}, H \longleftarrow H^{\text {op }}\right)$ 上一斜配对 $\sigma, B, H$ 的正则余模结构诱导张量积代数 $B \longleftarrow H$ 上一左 $\left(B \leftarrow B^{\text {cop }}\right.$, $\left.H \longleftarrow H^{\mathrm{op}}\right)$ 双余模代数结构, 相应的扭曲积 $(B \longleftarrow H)$ 。与 $B \longleftarrow H$ 的张量积余代数结构一起构成 Hopf 代数, 且 $A \sigma$ 仍是左 $(B \longleftarrow H)$ 。余模代数. 特别地, 从扭曲观点描述了 Drinfeld 量子偶. 另 外, 某些 Smash 积构作亦是以上扭曲积的特殊情形. 对偶地, 从斜余配对出发引入扭曲余积 构作, 描述了 Drinfeld 余量子偶和某些 Smash 余积.

\section{关键词 斜( 余)配对 扭曲( 余) 积 (余)量子偶}

任一有限维 Hopf 代数 $H$ 对应一个拟三角 Hopf 代数 $D(H)$-Drinfeld 量子偶, 作为余代 数, $D(H)=H^{*} \operatorname{cop} \longleftarrow H$, 其代数结构由一组交换关系给出 ${ }^{[1]}$. 按 Hopf 代数扩张观点, $D(H)$ 是一个双重 cross 积, 由 $H$ 与 $H^{*}$ cop彼此余伴随作用确定 ${ }^{[2]}$. 利用 $H^{*}$ 与 $H$ 间的标准配对两 次扭曲左 $H^{*}$ 右 $H$ 双余模代数 $H^{*} \longleftarrow H$ 即可得到 $D(H)^{\mathrm{op}[3]}$; 文献 [4] 给出了这一扭曲构作的 对偶形式. 本文从 $\left(H^{*}\right.$ cop,$\left.H\right)$ 上的斜配对出发, 建立一个新的扭曲定理, 更简单、直接地将 $H^{*}$ cop $4 H$ 一次扭曲得到 $D(H)$, 进一步, 得到扭曲积代数的一个一般性质, 也给出对偶情形 的扭曲构作, 直接得到 $D(H)$ * 的余积运算. 以下总设 $B, H$ 为域 $k$ 上的 Hopf 代数, 其对映映 射 $S_{B}, S_{H}$ 皆为双射; $H^{\mathrm{op}}, H^{\mathrm{cop}}$ 分别表示 $H$ 取反乘积、反余乘积所得 Hopf 代数; 未说明的记 号、概念参见文献 $[5,6]$.

\section{1 扭曲积和量子偶}

定义 1 称双线性映射 $\sigma: B \times H^{\rightarrow} k$ 为 $(B, H)$ 上一个(斜) 配对, $\forall a, b \in B, h, g \in$ $H$, 如果 $\sigma$ 满足以下条件:

$$
\begin{aligned}
& \sigma(1, h)=\varepsilon(h), \quad \sigma(b, 1)=\varepsilon(b) ; \\
& \sigma(a b, h)=\sum \sigma\left(a, h_{1}\right) \sigma\left(b, h_{2}\right) ; \\
& \sigma(a, h g)=\sum \sigma\left(a_{1}, h\right) \sigma\left(a_{2}, g\right) ; \\
& \sigma(a, h g)=\sum \sigma\left(a_{2}, h\right) \sigma\left(a_{1}, g\right) .
\end{aligned}
$$

例 1 若 $H$ 是有限维的, 定义 $\sigma: H^{*} \times H^{\rightarrow} k, \sigma(f, h)=f(h), \forall f \in H^{*}, h \in H$, 则 $\sigma$ 是 $\left(H^{*} ， H\right)$ 上的配对, 也是 $\left(H^{*}\right.$ cop,$\left.H\right)$ 上的斜配对. 一般, 若 $\sigma$ 是 $(B, H)$ 上一个配对, 则 
$\sigma$ 是 $\left(B^{\mathrm{op}}, H^{\mathrm{cop}}\right)$ 和 $\left(B^{\mathrm{cop}}, H^{\mathrm{op}}\right)$ 上的配对, 也是 $\left(B^{\mathrm{cop}}, H\right)$ 及 $\left(B, H^{\mathrm{op}}\right)$ 上的斜配对, 而 $\sigma_{21}$ 是 $(H, B)$ 上的配对, 这里 $\sigma_{21}(h, b)=\sigma(b, h), \forall b \in B, h \in H$.

设代数 $A$ 有左 $B$ 、左 $H$ 双余模结构而成为左 $B$ 余模代数和左 $H$ 余模代数, 等价地, $A$ 是 左 $B \longleftarrow H$ 余模代数, 也称 $A$ 为左 $(B, H)$ 双余模代数; 余模结构映射分别记为: $\Phi_{B}: A \rightarrow B \leftarrow$ $A,\left.a\right|^{\rightarrow} \sum_{a^{-1}} \leftarrow a^{0} ; \Phi_{H}: A \rightarrow H \leftarrow A,\left.a\right|^{\rightarrow} \sum_{a^{(-1)}} \leftarrow a^{(0)}$; 易见双余模条件可表为

$$
\sum a^{-1} \leftarrow a^{0(-1)} \leftarrow a^{0(0)}=\sum a^{(0)-1} \leftarrow a^{(-1)} \leftarrow a^{(0) 0}, \forall a \in A .
$$

定理 1 设 $\sigma$ 是 $(B, H)$ 上的斜配对, $A$ 是左 $(B, H)$ 双余模代数, 定义

$$
a \cdot b=\sum \sigma\left(b^{-1}, a^{(-1)}\right) a^{(0)} b^{0}, \forall a, b \in A .
$$

则 $(A, \cdot \sigma, 1)$ 仍是一个代数, 称为 $A$ 的扭曲积, 记为 $A \sigma$.

证 $\forall a, b, c \in A$, 由式(1), (5)得 $1 \cdot a a=a \cdot{ }_{\sigma} 1=a$, 且由式(2), (3'), (4), (5) 及余模代 数条件得 $(a \cdot b) \cdot c=a \cdot o(b \cdot a)$.

推论 $\mathbf{1}^{[3]}$ 若 $\sigma$ 是 $(B, H)$ 上的配对, $A$ 是左 $B$ 右 $H$ 双余模代数, 定义 $a \times b=\sum \sigma\left(a^{-1}\right.$, $\left.b^{(1)}\right) a^{0} b^{(0)}, \forall a, b \in A$, 则 $(A, \times, 1)$ 仍是一个代数, 这里右 $H$ 余模结构映射记为 $\Phi_{H}(a)$ $=\sum a^{(0)} \leftarrow a^{(1)}$.

证 $\sigma_{21}$ 是 $\left(H^{\mathrm{cop}}, B\right)$ 上的斜配对, $A$ 是左 $\left(H^{\mathrm{cop}}, B\right)$ 双余模代数, 其左 $H^{\mathrm{cop}}$ 余模结构由 $\Phi_{H}$ 诱导 $\Phi_{H}{ }^{\mathrm{cop}}(a)=\sum_{a^{(1)}} a^{(0)}$, 比较乘积公式得 $\left(A, \bullet_{\sigma_{21}}, 1\right)=(A, \times, 1)$.

若 $\sigma$ 是 $(B, H)$ 上的斜配对, $A$ 是左 $B$ 余模代数, $\forall h \in H, a \in A$, 令 $h \cdot a=\sum \sigma\left(a^{-1}\right.$, $h) a^{0}$, 易证 $(A, \cdot)$ 是左 $H$ 模且是 $H$ 模代数, 可作 Smash 积 $A \# H$; 另一方面, $\forall a \in A, h \in$

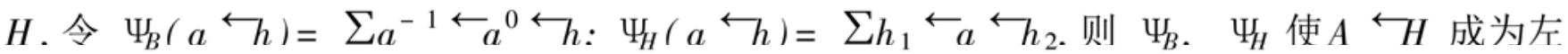
$(B, H)$ 双余模代数, 由定理 1 可作扭曲积 $(A \leftarrow H) \sigma$, 其乘积公式为 $(a \leftarrow h) \leftarrow_{\sigma}\left(b \leftarrow_{g}\right)=$ $\sum \sigma\left(b^{-1}, h_{1}\right)\left(a h_{2}\right)\left(b^{0} \leftarrow_{g}\right)=\sum_{a} \sigma\left(b^{-1}, h_{1}\right) b^{0} \bigsqcup_{2} g=\sum_{a}\left(h_{1} \bullet b\right) \leftarrow h_{2} g, \quad \forall a, b \in A$, $h, g \in H$, 于是证明了

推论 2 若 $\sigma$ 是 $(B, H)$ 上的斜配对, $A$ 是左 $B$ 余模代数, 则 $A$ 是左 $H$ 模代数, $A \longleftarrow H$ 是 左 $(B, H)$ 双余模代数, 且 $A \# H \cong(A \longleftarrow H)$.

仍设 $\sigma$ 是 $(B, H)$ 上的斜配对, 易见 $B$ 的正则左 $B$ 、右 $B$ 双余模代数结构诱导 $B$ 上, 因而 $B \longleftarrow H$ 上左 $B=B \longleftarrow B^{\mathrm{cop}}$ 余模代数结构: $\Phi_{B}(a \longleftarrow h)=\Sigma_{a_{1}} \leftarrow a_{3} \leftarrow a_{2} \longleftarrow h$; 类似地, $H$ 的左、右正 则双余模代数结构由斜对映映射转化为左 $H=H \longleftarrow H^{o p}$ 余模代数结构, 由此诱导 $(B \longleftarrow H)$ 上左 $H$ 余模代数结构: $\varphi_{H}(a \leftarrow h)=\sum h_{1} \leftarrow S_{H}^{-1} h_{3} \leftarrow a \leftarrow h, a \in A, h \in H$; 显然 $B \leftarrow H$ 是左 $(\boldsymbol{B}$, $\boldsymbol{H})$ 双余模代数. 另一方面, 容易验证 $\sigma$ 诱导 $(\boldsymbol{B}, \boldsymbol{H})$ 上一个斜配对 $\sigma: \sigma\left(b \leftarrow b^{\prime}, h \leftarrow h^{\prime}\right)=$ $\sigma(b, h) \sigma\left(b^{\prime}, h^{\prime}\right), \forall b \in B, b^{\prime} \in B^{c o p}, h \in H, h^{\prime} \in H^{o p}$. 由定理 1 , 作扭曲积 $(B \longleftarrow H) \sigma$, 易 见,

$$
(a \leftarrow h) \cdot \sigma(b \leftarrow g)=\sum \sigma\left(b_{1}, h_{1}\right) a b_{2} \leftarrow h_{2} g \sigma\left(b_{3}, S_{H}^{-1} h_{3}\right), \quad \forall a, b \in B, h, g \in H,
$$

注意到 $\sigma$ 可逆且 $\sigma^{1}=\sigma\left(i d \leftarrow S_{H}^{-1}\right)$, 直接验证知, $(B \leftarrow H)$ 。的张量积余代数结构映射 $\Delta \varepsilon$ 是 代数映射, $S(a \longleftarrow h)=\sum \sigma\left(S_{B} b_{3}, S_{H} h_{3}\right) S_{B} b_{2} \leftarrow S_{H} h_{2} \sigma^{-1}\left(S_{B} b_{1}, S_{H} h_{1}\right)$ 为 $(B \leftarrow H)$ 。的对映公 式. 
定理 2 若 $\sigma$ 为 $(B, H)$ 上的斜配对, 则( ) 按以上定义, $B \longleftarrow H$ 是左 $(B, H)$ 双余模代 数, $\boldsymbol{\sigma}$ 是 $(\boldsymbol{B}, \boldsymbol{H})$ 上的斜配对, 且扭曲积代数 $(B \longleftarrow H) \sigma$ 与其张量积余代数一起构成 Hopf 代数; ( ) 若 $A$ 是左 $B \longleftarrow H$ 余模代数, 则 $A \sigma$ 是左 $(B \longleftarrow H)$ 。余模代数.

证 ( ) 已证. ( ) $A$ 是左 $B \longleftarrow H$ 余模代数, 其余模结构映射为: $\forall a \in A, \Phi(a)=$

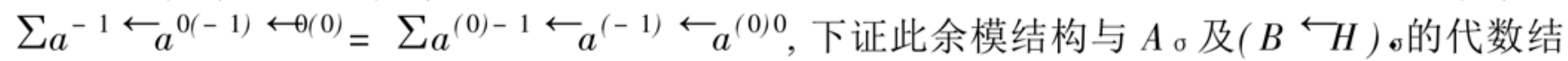
构相容: $\forall a . b \in A$.

$$
\begin{aligned}
\Phi(a) \cdot \Phi(b)=\sum\left(a^{(0)-1} \leftarrow a^{(-1)}\right) \cdot \sigma\left(b^{-1} \leftarrow b^{0(-1)}\right) \leftarrow a^{(0) 0} \cdot b^{0(0)} \\
\frac{5,6}{} \sum \sigma\left(b_{1}^{-1}, a_{1}^{(-1)}\right) a^{(0)-1} b_{2}^{(-1)} \leftarrow a_{2}^{-1} b^{0(-1)} \sigma^{1}\left(b_{1}^{-1}, a_{3}^{(-1)}\right) \\
\leftarrow \sigma\left(b^{0(0)-1}, a^{(0) 0(-1)}\right) a^{(0) 0(0)} b^{0(0) 0} \\
\frac{4}{} \sum \sigma\left(b_{1}^{-1}, a_{1}^{(-1)}\right) a^{(0)-1} b_{2}^{-1} \leftarrow a_{2}^{(-1)} b^{0(-1)} \sigma^{1}\left(b_{3}^{-1}, a_{3}^{(-1)}\right) \\
\leftarrow \sigma\left(b_{4}^{-1}, a_{4}^{(-1)}\right) a^{(0) 0} b^{0(0)} \\
=\sum \sigma\left(b_{1}^{-1}, a_{1}^{(-1)}\right) a^{(0)-1} b_{2}^{-1} \leftarrow a_{2}^{(-1)} b^{0(-1)} \leftarrow a^{(0) 0} b^{0(0)} \\
=\sum \sigma\left(b^{-1}, a^{(-1)}\right) a^{(0)(0)-1} b^{0-1} \leftarrow a^{(0)(-1)} b^{00(-1)} \leftarrow a^{(0)(0) 0} b^{00(0)} \\
=\sum \sigma\left(b^{-1}, a^{(-1)}\right)\left(a^{(0)} b^{0}\right)^{-1} \leftarrow\left(a^{(0)} b^{0}\right)^{0(-1)} \leftarrow\left(a^{(0)} b^{0}\right)^{0(0)}=\Phi(a \cdot b) .
\end{aligned}
$$

推论 3 若 $H$ 为有限维 Hopf 代数, 则 $D(H)=\left(H^{*} \operatorname{cop} \longleftarrow H\right)$, 其中 $\sigma$ 为赋值映射.

证 $\forall f, g \in H^{* \text { cop }}, a, b \in H$, 在 $D(H)$ 中, $(f \leftarrow a)(g \leftarrow b)=\sum f\left(a_{1}-g-S_{H}^{-1} a_{3}\right) \leftarrow$ $a_{2} b=\sum \sigma\left(g_{3}, a_{1}\right) f g_{2} \leftarrow a_{2} b \sigma\left(g_{1}, S_{H}^{-1} a_{3}\right)^{[6]}$, 注意到 $\Delta_{B}(g)=\sum_{g_{2}} \leftarrow g_{1}$, 与式(6) 比较知与

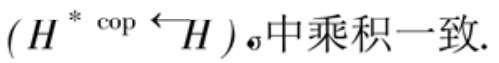

\section{2 对偶情形}

定义 2 称 $R=\sum R^{(1)} \leftarrow R^{(2)} \in B \longleftarrow H$ 为 $(B, H)$ 的一个(斜) 余配对, 如果 $R(R=r)$ 满足 以下条件:

$$
\begin{gathered}
\sum \varepsilon\left(R^{(1)}\right) R^{(2)}=1_{H}, \quad \sum R^{(1)} \varepsilon\left(R^{(2)}\right)=1_{B}, \\
\sum R_{1}^{(1)} \leftarrow R_{2}^{(1)} \leftarrow R^{(2)}=\sum R^{(1)} \leftarrow r^{(1)} \leftarrow R^{(2)} r^{(2)}, \\
\sum R^{(1)} \leftarrow R_{1}^{(2)} \leftarrow R_{2}^{(2)}=\sum R^{(1)} r^{(1)} \leftarrow R^{(2)} \leftarrow{ }^{(2)} \\
\sum R^{(1)} \leftarrow R_{1}^{(2)} \leftarrow R_{2}^{(2)}=\sum R^{(1)} r^{(1)} \leftarrow r^{(2)} \leftarrow R^{(2)} .
\end{gathered}
$$

若 $H$ 为有限维 Hopf 代数, $\left\{h_{i}\right\}_{i=1}^{n},\left\{h_{i}^{*}\right\}_{i=1}^{n}$ 为 $H$ 和 $H^{*}$ 的对偶基, 令 $R=\sum_{i=1}^{n} h_{i} \longleftarrow h_{i}^{*}$, 则 $R$ 是 $\left(H^{\mathrm{op}}, H^{*}\right)$ 的斜余配对, 是 $\left(H, H^{*}\right)$ 的余配对. 一般, 若 $R$ 为 $(B, H)$ 的一个余配对, 则 它也是 $\left(B^{\mathrm{op}}, H\right)$ 及 $\left(B, H^{\mathrm{cop}}\right)$ 的斜余配对, 也是 $\left(B^{\mathrm{op}}, H^{\mathrm{cop}}\right)$ 和 $\left(B^{\mathrm{cop}}, H^{\mathrm{op}}\right)$ 的余配对, 而 $R_{21}=$ $\sum R^{(2)} \longleftarrow R^{(1)}$ 是 $(H, B)$ 的余配对. 称有左 $B$ 、左 $H$ 双模结构而成为左 $B$ 模余代数和左 $H$ 模 余代数的余代数 $C$ 为左 $(B, H)$ 双模余代数, 等价地, $C$ 是左 $B \longleftarrow H$ 模余代数, 用同一符号“・” 表示 $C$ 的左 $B, H$ 及诱导的左 $B \longleftarrow H$ 作用不致引起混乱, 且将 $B$ 等同 $B \longleftarrow \subset B \longleftarrow H(H=1 \leftarrow$ $H)$, 则有:

$$
b \cdot(h \cdot c)=h \cdot(b \cdot c)=b h \cdot c=h b \cdot c, \forall b \in B, c \in C, h \in H .
$$


定理 3 设 $R$ 是 $(B, H)$ 的一个斜余配对, $C$ 是左 $(B, H)$ 双模余代数, 定义

$$
\Delta_{R}(c)=\sum R^{(2)} \cdot c_{1} \leftarrow R^{(1)} \cdot c_{2}, \forall c \in C,
$$

则 $C_{R}=\left(C, \Delta_{R}, \varepsilon\right)$ 仍是余代数, 称为 $C$ 的扭曲余积.

证 由式(7)，(8)，(9')，(10)，(11) 及模余代数条件得证.

推论 $4^{[4]}$ 若 $R$ 是 $(B, H)$ 的余配对, $C$ 为左 $B$, 右 $H$ 双模余代数, $\forall c \in C$, 定义 $\nabla(c)=$ $\sum R^{(1)} \cdot c_{1} \leftarrow c_{2} \cdot R^{(2)}$, 则 $(C, \Omega(\varepsilon)$ 仍为余代数.

推论 5 设 $R$ 是 $(B, H)$ 的斜余配对, $C$ 是左 $B$ 模余代数, $\forall c \in C$, 令 $\Phi(c)=\sum R^{(2)} \leftarrow$ $R^{(1)} \cdot c$, 则 $\Phi$ 使 $C$ 成为左 $H$ 余模余代数; 令 $b \cdot(c \leftarrow h)=b \cdot c \leftarrow h, g \cdot(c \leftarrow h)=c \leftarrow g h, \forall b \in$ $B, c \in C, h, g \in H$, 则 $C \longleftarrow H$ 是左 $(B, H)$ 双模余代数, 且 smash 余积 $C \times H \cong(C \longleftarrow H)_{R^{-}}$扭 曲余积.

定理 4 设 $R$ 是 $(B, H)$ 的斜余配对, 则 ( ) $B, H$ 的正则双模余代数结构诱导 $B \leftarrow H$ 的 一个左 $\left(B \leftarrow B^{\text {cop }}, H \longleftarrow H^{\text {op }}\right)$ 双模余代数结构: $(c \longleftarrow d) \cdot(b \longleftarrow)=c b S_{B} d \longleftarrow,(g \leftarrow h) \cdot(b \longleftarrow)=$ $b \leftarrow_{g} l h, \forall b, c, d \in B, g, l, h \in H, R=\sum R^{(1)} \leftarrow_{r}^{(1)} \leftarrow_{R}^{(2)} \leftarrow_{r}^{(2)}(R=r)$ 是 $\left(B \leftarrow B^{\text {cop }}\right.$, $\left.H \longleftarrow H^{\mathrm{op}}\right)$ 上的一个斜余配对, 且扭曲余积 $(B \longleftarrow H)_{R}$ 与其张量积代数结构一起构成 Hopf 代数; ( ) 若 $C$ 是左 $(B, H)$ 双模余代数, 则 $C_{R}$ 是左 $(B \leftarrow H)_{R}$ 模余代数.

证（）易见 $\forall a, b \in B, h \in H ， \Delta_{R}(b \leftarrow h)=\sum b_{1} \leftarrow R^{(2)} h_{1} r^{(2)} \leftarrow_{R}^{(1)} b_{2} S_{B} r^{(1)} \leftarrow_{2}$; $S(b \longleftarrow h)=\sum S_{B}\left(r^{(1)} b R^{(1)}\right) \leftarrow R^{(2)} S_{H}\left(r^{(2)} h\right)$, 细节略. ( ) 由条件知 $C$ 是左 $B \leftarrow H$ 模余代 数. 注意到 $R^{-1}=\sum R^{(1)} \leftarrow S_{H}^{-1} R^{(2)}=\sum S_{B} R^{(1)} \leftarrow R^{(2)}, \forall b \in B, c \in C, h \in H, \Delta_{R}(b \leftarrow h) \bullet$ $\Delta_{R}(c) \stackrel{11}{\longleftarrow} \Sigma\left(b_{1} \leftarrow R^{(2)} h_{1} r^{(2)}\right) \cdot\left(\boldsymbol{R}^{(2)} \cdot c_{1}\right) \leftarrow\left(R^{(1)} b_{2} S_{B} r^{(1)} \leftarrow h_{2}\right) \cdot\left(\boldsymbol{R}^{(1)} \cdot c_{2}\right) \frac{10}{\longleftarrow} \Sigma\left(b_{1} \leftarrow R^{(2)}\right.$ $\left.\cdot h_{1} r^{(2)} \boldsymbol{R}^{(2)}\right) \cdot c_{1} \leftarrow\left(R^{(1)} b_{2} S_{B} r^{(1)} \boldsymbol{R}^{(1)} \leftarrow_{2}\right) \cdot c_{2}=\sum R^{(2)} h_{1} \cdot b_{1} \cdot c_{1} \leftarrow R^{(1)} b_{2} \cdot h_{2} \cdot c_{2}=\sum R^{(2)} \cdot$ $\left(\left(b_{1} \leftarrow_{1}\right) \cdot c_{1}\right) \leftarrow R^{(1)} \cdot\left(\left(b_{2} \leftarrow_{2}\right) \cdot c_{2}\right)=\sum R^{(2)} \cdot((b \leftarrow h) \cdot c)_{1} \leftarrow R^{(1)} \cdot((b \leftarrow h) \cdot c)_{2}=\Delta_{R}((b$ $\leftarrow$ h) $c$ c)，其中 $R=R=r$.

推论 6 若 $H$ 是有限维 Hopf 代数, 则 $D(H)^{*} \cong\left(H^{\text {op }} \leftarrow^{*}\right)_{R}$, 其中 $R=\sum_{i=1}^{n} h_{i} \leftarrow h_{i}^{*}$, $\left\{h_{i}\right\},\left\{h_{i}^{*}\right\}$ 是 $H$ 和 $H^{*}$ 的对偶基.

致谢 本工作为广东省博士后基金及国家自然科学基金(批准号: 19771090)资助项目.

\section{参 考 文 献}

1 Drinfeld V G. Quantum Groups. In: Proc ICM. Berkeley: AMS, 1997. 789 820

2 Majid S. Physics for algebraists. J Alg, 1990, 130: 17 64

3 Santos W R F. Twisting products in Hopf algebras and the construction of the quantum double. Comm Alg, 1995, 23(7): 789 820

4 赵文正, 王栓宏, 焦争鸣. 扭曲余积和 $H^{R}$ 型 Hopf 代数. 数学学报, 1997, 40(4) : 591 596

5 Sw eedler M E. Hopf Algebras. New York: Benjamin, 1969. 1 30

6 Montgomery S. Hopf algebras and their actions on rings. CBMS LN 82. Providence: AMS, 1993 